\title{
What is the role of cardiac MRI in the diagnosis of left ventricular non-compaction?
}

\author{
J Ronald Mikolich ${ }^{1,2^{*}}$, John Lisko ${ }^{1}$, Nicholas C Boniface ${ }^{2}$, Brandon M Mikolich ${ }^{1,2}$ \\ From 16th Annual SCMR Scientific Sessions \\ San Francisco, CA, USA. 31 January - 3 February 2013
}

\section{Background}

The prevalence of left ventricular non-compaction is not well established. Using 2-D echocardiography, less than $1 \%$ of patients are diagnosed with this entity. Using cardiac MRI, approximately $3 \%$ of patients have evidence of LV non-compaction. This variance may be related to the difference in spatial resolution between these 2 imaging techniques. Using a large institutional cardiac imaging database, 2-D echo and cardiac MRI results were compared in patients with LV non-compaction.

\section{Methods}

Of 1,255 patients in our cardiac imaging database, 38 patients $(3 \%)$ with a diagnosis of LV non-compaction were identified. Twenty-two of these 38 patients had undergone both 2-D echo and cardiac MRI exams. The reported diagnoses with each imaging modality were tabulated, along with measurements of left ventricular antero-septal and infero-posterior wall thickness for both modalities. The 2-D echo and cardiac MRI wall thickness dimensions were statistically compared using a paired sample t-test.

\section{Results}

All 38 patients had a diagnosis of LV non-compaction on cardiac MRI, using criteria of non-compacted/compacted myocardium $>2.5$ to 1.0 and deep LV trabeculations. None of the 22 patients with both 2-D echo and cardiac MRI exams had a diagnosis of LV non-compaction on their 2-D echo study. However, 15 of 22 patients (68\%) had a diagnosis of LVH on their 2-D echo study, while only 3 had LVH on their cardiac MRI study. The mean ASWT on 2-D echo was $1.38 \mathrm{~cm}$, while the mean ASWT on cMRI was $1.16 \mathrm{~cm}(\mathrm{p}<0.005)$. The mean IPWT on 2-D echo was $1.31 \mathrm{~cm}$, while the IPWT on cMRI was only 0.89 $\mathrm{cm}(\mathrm{p}<0.001)$.

\section{Conclusions}

These data suggest that LV non-compaction, identifiable on cardiac MRI, is frequently diagnosed as LVH on 2-D echo. This discrepancy appears to be explained by the statistically significant difference in both antero-septal and infero-posterior LV wall thickness measurements between the 2 imaging modalities. It is suspected that this difference is related to the inferior spatial resolution of 2-D echo relative to cMRI. On the basis of these data, patients with LVH on 2-D echo should be considered for a cardiac MRI exam to determine if the increased LV wall thickness is truly LVH or an undiagnosed LV non-compaction cardiomyopathy.

\section{Funding}

Not Applicable. None.

\section{Author details \\ ${ }^{1}$ Northeast Ohio Medical University, Rootstown, OH, USA. ${ }^{2}$ Sharon Regional Health System, Hermitage, PA, USA.}

Published: 30 January 2013

doi:10.1186/1532-429X-15-S1-P157

Cite this article as: Mikolich et al:. What is the role of cardiac MRI in the diagnosis of left ventricular non-compaction? Journal of Cardiovascular Magnetic Resonance 2013 15(Suppl 1):P157.

${ }^{1}$ Northeast Ohio Medical University, Rootstown, OH, USA

Full list of author information is available at the end of the article

(c) 2013 Mikolich et al; licensee BioMed Central Ltd. This is an Open Access article distributed under the terms of the Creative Commons Attribution License (http://creativecommons.org/licenses/by/2.0), which permits unrestricted use, distribution, and reproduction in any medium, provided the original work is properly cited. 